\section{OC-107 HELICOBACTER PYLORI INDUCES HUMAN DENDRITIC CELL MATURATION AND DUODENAL ULCER PROMOTING GENE A (DUPA) ENHANCES DENDRITIC CELL CYTOKINE EXPRESSION}

doi:10.1136/gut.2011.239301.107

M Z Cader, ${ }^{*}$ C Marx, E Staples, K Robinson, J Atherton Nottingham Digestive Diseases Centre, University of Nottingham, Nottingham, UK

Introduction Helicobacter pylori is the leading cause of peptic ulceration worldwide and possession of duodenal ulcer promoting gene $\mathrm{A}(\mathrm{dup} A)$ increases ulcer risk. In vitro, dupA increases production of pro-inflammatory cytokines by monocytes, but these cells are not present in gastric mucosal tissue. However, isolation and characterisation of immune cells from human gastric biopsy samples has shown the presence of myeloid dendritic cells. Dendritic cells are antigen-presenting cells with a unique ability to initiate a primary immune response from naïve T-cells. We therefore investigated whether Helicobacter pylori is able to induce dendritic cell maturation and pro-inflammatory cytokine expression and whether this is influenced by the dupA gene.

Methods Human dendritic cells were derived from circulating monocytes from healthy donors. These cells were co-cultured with $H$ pylori strains AB21 and AB31 and their isogenic dupA knockout mutants. The activation status of dendritic cells was assessed by flow cytometry; cytokine production was quantified by ELISA.

Results In their resting state the monocyte derived dendritic cells expressed MHC class II, but very low levels of co-stimulatory molecules CD80, CD83 and CD86. Upon culture with $H$. pylori, the cells expressed significantly higher levels of these co-stimulatory molecules, demonstrating maturation $>25$ fold increase in percentage of positive events for CD80, CD83, CD86 on flow cytometry; $p<0.01)$. There were no differences between the responses to wild type and dupA knockout mutant strains, or following stimulation with lipopolysaccharide (LPS). The $H$. pylori-matured dendritic cells secreted high levels of IL-12p40, IL-12p70, IL-10 and IL-23. The concentrations induced by the dupA+ strains were significantly higher than those induced by the dupA mutants ( 1.5 fold increase in IL-12p40 production, $\mathrm{p}<0.05 ; 1.4$ fold increase in IL-12p70, $\mathrm{p}<0.05)$.

Conclusion $H$ pylori induces dendritic cell maturation and secretion of pro-inflammatory cytokines. dupA increases IL-12 production from these dendritic cells. This may explain the enhanced antral gastritis and ulcer risk associated with dupA + H pylori strains.

Competing interests None.

Keywords duodenal ulcer promoting gene A, Helicobacter pylori. 